\title{
Development of Sensibility Vocabulary Classification System for Sensibility Evaluation of Visitors According to Forest Environment
}

\author{
Jeong-Do Lee, Dawou Joung, Sung-Jun Hong, Da-Young Kim, and Bum-Jin Park ${ }^{*}$ \\ Department of Environment \& Forest Resources, Chungnam National University, Daejeon 34134, Korea
}

\begin{abstract}
Generally human sensibility is expressed in a certain language. To discover the sensibility of visitors in relation to the forest environment, it is first necessary to determine their exact meanings. Furthermore, it is necessary to sort these terms according to their meanings based on an appropriate classification system. This study attempted to develop a classification system for forest sensibility vocabulary by extracting Korean words used by forest visitors to express their sensibilities in relation to the forest environment, and established the structure of the system to classify the accumulated vocabulary. For this purpose, we extracted forest sensibility words based on literature review of experiences reported in the past as well as interviews of forest visitors, and categorized the words by meanings using the Standard Korean Language Dictionary maintained by the National Institute of the Korean Language. Next, the classification system for these words was established with reference to the classification system for vocabulary in the Korean language examined in previous studies of Korean language and literature. As a result, 137 forest sensibility words were collected using a documentary survey, and we categorized these words into four types: emotion, sense, evaluation, and existence. Categorizing the collected forest sensibility words based on this Korean language classification system resulted in the extraction of 40 representative sensibility words. This experiment enabled us to determine from where our sensibilities that find expressions in the forest are derived, that is, from sight, hearing, smell, taste, or touch, along with various other aspects of how our human sensibilities are expressed such as whether the subject of a word is person-centered or object-centered. We believe that the results of this study can serve as foundational data about forest sensibility.
\end{abstract}

Keywords: emotion, forest environments, national language adjective, sensibility words

\section{Introduction}

As the field of study on sensibility expanded to all industrial fields from psychology to engineering as well as information technology (IT) and biology technology (BT), there has been an increasing need for vocabulary data related to expression of sensibility (Sohn et al., 2012). Sensibility vocabulary is mostly classified as qualitative adjectives in Korean language, which is because most adjectives that reveal human sensibility are qualitative adjectives (Sin, 1996). Qualitative adjectives refer to the properties and state of objects or properties and behaviors of people, and vocabulary about the

This study is supported by the forest science technology R\&D project (2016003C10-1619-AB01) of the Korea Forestry Promotion Institute under the Korea Forest Service.

Received: January 17, 2019, Revised: February 9, 2019, Accepted: February 21, 2019

First author: Jeong-Do Lee, E-mail: volom19@gmail.com, (1) https://orcid.org/0000-0002-3207-5969

*Corresponding author: Bum-Jin Park, E-mail: bumjinpark.cnu@gmail.com, (1) https://orcid.org/0000-0002-5214-5688

2019 by the Society for People, Plants, and Environment. This is an Open Access article distributed under the terms of the Creative Commons Attribution Non-Commercial License (http://creativecommons.org/licenses/by-nc/4.0/) which permits unrestricted non-commercial use, distribution, and reproduction in any medium, provided the original work is properly cited. 
characteristics of seongsang (quality) have been studied by many Korean linguists (Choi, 1937; Hong, 1985; Nam and Ko, 1985; Lee and Lee, 1990; Son, 1992; Sin, 1996; Lee, 1998; Yoo, 2000; Jang, 2001). Recently, the application scope of sensibility vocabulary is further expanding for purposes such as product design or selection of building colors (Jung and Nah, 2007; Lee and Kim, 2013). This is due to the improvement of living standards and stabilization of the five-day workweek system. With people spending more and more time enjoying leisure and culture, their value system about the quality of life has changed qualitatively (Lee et al., 2019). Keeping pace with such change, Korea Forest Service is constantly building new healing forests or recreational forests to promote national welfare, and pursuing the strategic goal of providing high quality forest welfare services through Korea Forest Welfare Institute (Korea Forest Service, 2017; Korea Forest Welfare Institute, 2017). This shows the intent to increase recreational facilities for national welfare and provide various recreational spaces in terms of quality. Studies on forest sensibility will perform a crucial role in determining customer needs in such qualitative change. This is because the sensibility preference of visitors can be determined by revealing what sensibility they feel depending on the forest environment. Accordingly, the objective of this study is to develop a forest sensibility vocabulary classification system in order to determine human sensibility in forests with discrimination and objectivity. Thus far, there have been many studies on sensibility vocabulary, but they have a few limitations in using them according to forest sensibility vocabulary. First, the meaning of emotion words is limited to emotions such as 'pleasant-unpleasant' rather than individual emotions (Sohn et al., 2012). Second, the scope of sensibility vocabulary is broad and there were many words not related to forests. Third, researchers showed different views about semantic interpretation of sensibility words and classification criterion, making it difficult to quantitatively distinguish the words. Thus, this study extracted only the words that are relevant to forests among the extensive scope of sensibility vocabulary to organize a Korean vocabulary classification system. In addition, these two were linked to categorize the forest sensibility vocabulary by meaning. By doing so, this study will reveal the types of human sensibility experienced in forests and what factors are causing such sensibility.

\section{Data and Methods}

\section{Development process of the forest sensibility vocabulary classification system}

To develop the sensibility vocabulary classification system and extract vocabulary, this study first collected sensibility vocabulary related to forests based on literature review of publications (seven volumes), collection of experiences (one volume) and reports (one volume) covering experiences or interviews with forest visitors. Second, the Korean vocabulary classification system was examined with focus on 'qualitative adjectives.' Third, we linked the Korean adjective classification system and forest sensibility vocabulary, and integrated or excluded redundant words, meanings and antonyms in the process. Fourth, a Delphi survey was conducted on forest experts for words that remain after a series of selection processes, and we ultimately determined whether the collected forest sensibility words shall be accepted within the classification system in the perspective of forest experts (Figure 1).

\section{Investigation of the forest sensibility vocabulary and Korean vocabulary classification system}

To collect sensibility vocabulary of forest visitors, we used Eco Healing 2014-2015 Volumes 1-7 that mostly cover interviews or articles about forest welfare, '2014 Collection of Award-winning Works on Forest Healing Experiences' and 'Development and Use of the Forest Quality Assessment Method' published by National Institute of Forest Science (Korea Forest Service, 2012, 2014, 2014-2015). 
Literature review of

emotional vocabulary

in relation to forest
Organizing

classification system

of Korean adjectives
Connecting DB of Korean

adjective and forest

expressing vocabulary
Forest expert group

Delphi technique

Figure 1. Development process of the forest sensibility vocabulary classification system.

The Korean vocabulary classification system serves as a 'tool' necessary for forest sensibility vocabulary to have discrimination and objectivity and to avoid redundant use of similar meaning. To this end, it is necessary to examine previous studies on the Korean vocabulary classification system of scholars of Korean language and literature. Yoo (2000) categorized the classification system by the subject thematic role of adjectives. When the thematic role of the subject is undergoer, the adjective is classified as subjective adjective; when the thematic role of the subject is object/place, it is classified as objective adjective. These two groups are then subdivided into 12 categories according to case frame and syntactic and semantic characteristics. When the thematic role of the subject is undergoer, the adjectives are classified into 'psychology, decision, sense adjectives'; when the thematic role of the subject is object/place, they are classified into 'seongsang (quality), place, symmetry, criterion, possession, whereabouts, possibility, answer, attitude.' Son (1992) organized the classification system of existing researchers on sense adjectives and came up with a new system, and defined that humans must be the most fundamental discrimination criterion for sense adjectives. This must be based on the sensory qualities of body parts that have power of discrimination, such as the outside of the body like 'eyes, ears, nose, tongue, skin' and inside of the body like organic sensation and sense of balance, and whether there is a conflict also serves as a crucial discrimination criterion in systemizing the characteristics. Son (1992) also divided the classification system of sense adjectives into external and internal sense, and subdivided them into seven categories of sight, hearing, smell, taste, somatosensory sense, organic sensation and sense of balance.' For external sense, sight is classified into 'light, color, space,' hearing into 'sound (loud), tune (high or low), smell into 'scent,' taste into 'flavor,' and somatosensory sense into 'tough (rough, hard, thick), apperception (pain), temperature (hot and cold).' Internal sense is classified into organic sensation (breath, digestion, excretion) and sense of balance (vertigo). Sin (1996) divided the emotional adjective classification system into two groups: psychology adjectives and attribute adjectives. Here, psychology is not limited to a specific phenomenon or situation, but includes all psychological phenomena, which are categorized into sense and emotion (Lee, 1976). Attributes are characteristics or fundamental properties of people of objects, and most of them are objective attributes that are categorized into evaluation and existence. More specifically, sense vocabulary is categorized into five senses of humans such as 'sight (color, brightness, purity), hearing, smell, taste (basic, comprehensive, convertgence), touch (surface, apperception, temperature).' Emotion vocabulary is categorized into four emotions such as 'joy and sorrow/anger, preference, satisfaction/dissatisfaction, fear/trust,' and evaluation vocabulary is categorized into eight types of evaluation such as 'authenticity, right or wrong, good or evil, noble or lowly, beauty or ugliness, difficulty, profit, comparison.' Finally, existence vocabulary is categorized into 5 types of existence such as 'time (speed), space (existence, distance, depth, breadth and narrowness, density, layer), shape (length, high or low, size, weight, round or angled, thick or thin, sharp and dull, balance), quantity, behavior (free/busy, prudence, completeness, diligence, robustness, privacy).' We developed the forest sensibility vocabulary classification system based on the classification system by Sin (1996) among the vocabulary classification systems by scholars of Korean language and literature. This is because the system is classified into psychology and attribute factors, and is thus most suitable for structuring forest sensibility vocabulary and it is possible to determine which factors affect sensibility expressions in detailed categories. However, the emotion part of Sin 
(1996) has relatively fewer words than the investigated number of forest sensibility words, and thus was supplemented by emotion verbs by Hong and Jeong (2009). Hong and Jeong (2009) summarized previous studies by scholars of Korean language and literature on emotion vocabulary, categorizing 258 verbs into 19 emotion types such as 'impression, gratitude, anguish, loneliness, pleasure, fright, sympathy, fear, sorry, anger, fresh, accept, shame, sad, desire, relaxation, futility, hatred, regret'(Figure 2).

\section{Development of the forest sensibility vocabulary classification system}

In the process of connecting the Korean vocabulary classification system and forest sensibility vocabulary, we selected redundant words, redundant meanings and antonyms. Examples of redundant words were 'pureuda, pureun, pureureun' that all mean 'blue/green,' and examples of redundant meanings were 'chomchomhan, ppaekppaekhan, wugeojin' that all mean 'dense.' Accordingly, we selected one word with representativeness when there were different words that share the same meaning. Antonyms were opposite words such as 'bright-dark, familiar-unfamiliar, fresh-unpleasant.' Antonyms can be added in the final sensibility vocabulary classification system and thus in the connection process we selected on word with representativeness. A Delphi survey was conducted on forest experts for words that remained even after the series of selection processes. The Delphi method is conducting a survey on a panel of experts by meeting multiple times, and the experts comprised of 11 male and female graduate students (32.3 \pm 6.6 ) majoring or working in the field of forestry.

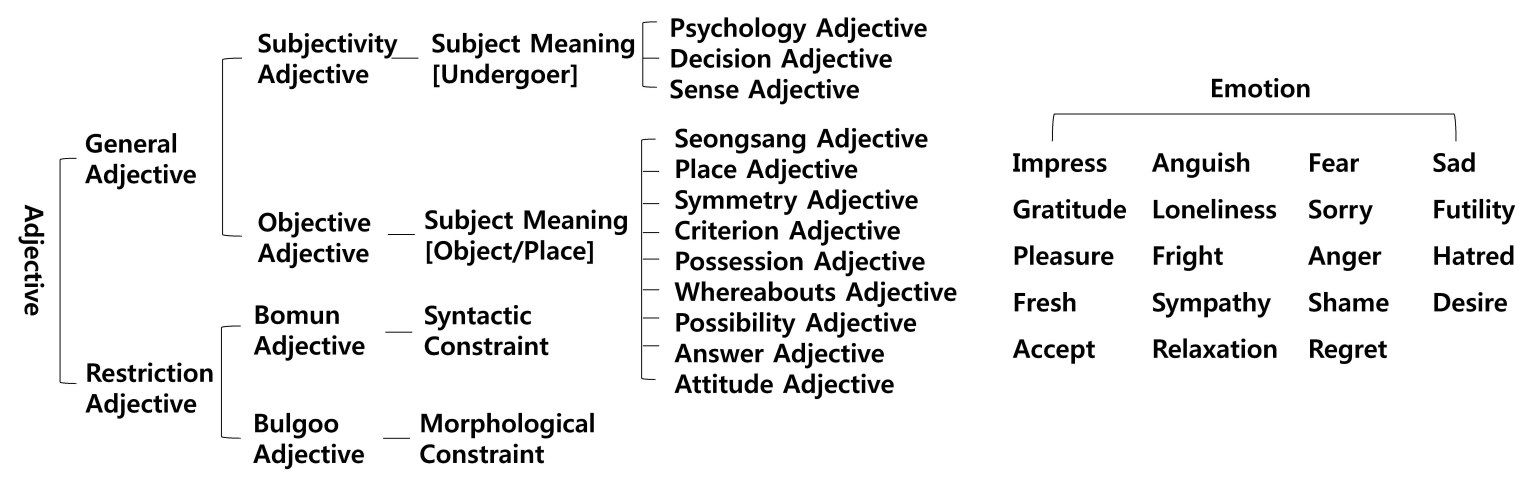

Yoo, H.K's (2000) Adjective Classification System

Hong, J.S`s (2009)Emotional Verb Classification System
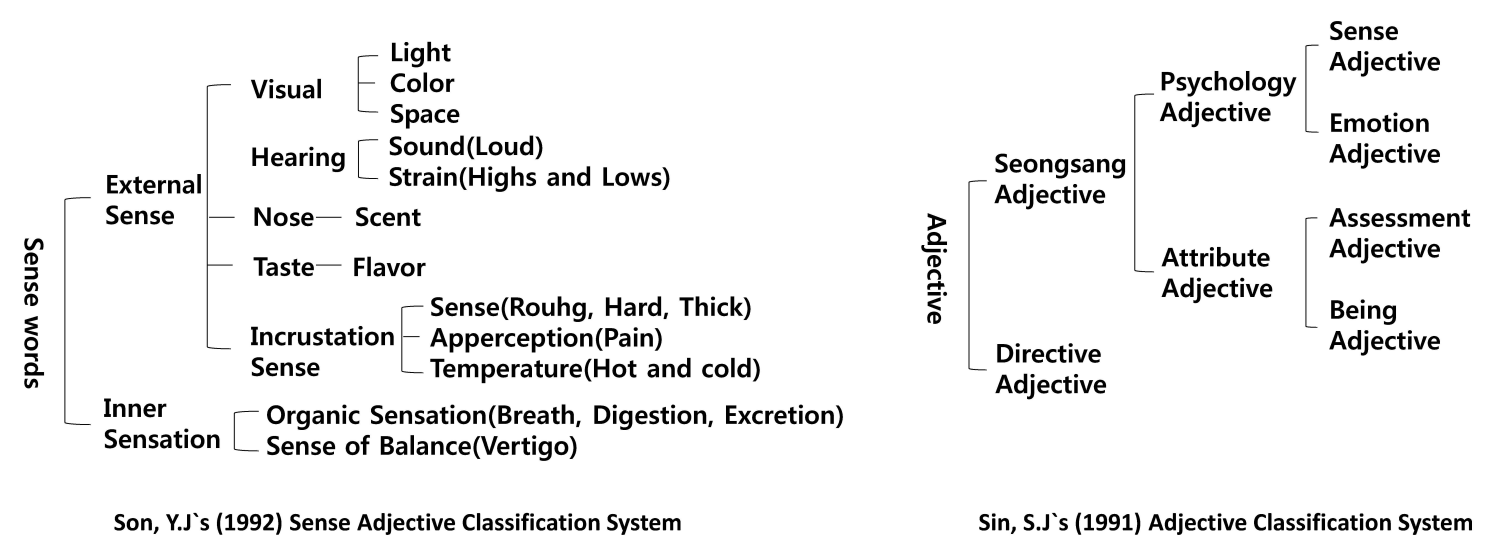

Sin, S.J`s (1991) Adjective Classification System

Figure 2. Different adjective classification systems used by Korean linguists. 
In the first survey, the experts were to select whether the remaining words belong to the 'sense, emotion, evaluation, existence' category of the Korean language classification system, and words with less than $50 \%$ response rate were eliminated, and those with at least 50\% response rate were selected. In the second survey, the experts selected words that can be replaced among the words with similar meanings found in the first survey. The Delphi method is used to determine whether the forest sensibility words examined must be accepted in the perspective of forest experts, thereby preventing meaningless elimination of words. This selection process is carried out with reference to the Standard Korean Language Dictionary of National Institute of the Korean Language (Standard Korean Dictionary, 2018).

\section{Results and Discussion}

Total 137 sensibility words were extracted, excluding those expressing tendencies or behaviors that are not sensibility or emotion in the literature review related to forest welfare. The words listed by frequency are as follows: 'good, comfortable (pyeonanhan), beautiful (areumdaun), clean (malgeun), happiness, fresh (sang kwaehan), stabile, enjoyable, dense (ulchanghan), blue (pureun), arcane, cool, warm (ttatteutan), excited, fresh (singgeureoun), leisurely, thankful, valuable, tough, wonderful, refreshing, pretty, clear, clean (kkaekkeutan), attractive, flutter, snug (pogeunhan), abundant (pungyoroun), healthy, silent, dense (ppaekppaekhan), funny, silent, peaceful (pyeonghwaroun), fancy, refreshed, various, lush, calm (chabunhan), peaceful (pyeonganhan), quiet (pyeongonhan), interesting, glad, soft (budeureoun), new, safety, jeonggyeoun, moist, abundant (pungseonghan), convenient, unchangeable, old, light, deep, amazing (nollaun), dazzling, friendly, nice, bright, inconvenient, an armful, grand, great (widaehan), delicate, cold (chagaun), cosy, big, peace, warm (pugeunhan), quiet (hojeothan), recover, mellow (gammiroun), appreciation, modesty, arduous, straight, great (goengjanghan), romantic, unfamiliar, high, varied, great (daedanhan), warm (ttaseuhan), trembling, treach, soft (bodeuraun), fruitful, full, fresh (santteuthan), animated, beautiful (suryeohan), amazing (singihan), charming, snug (aneukhan), dizzy, comfort, colorful, bright, demulcent, pleasant, familiar, fine, natural, free, calm (janjanhan), hefty, harmonious, rare (jingwihan), subside, grumous, racy, sunshiny, liquid, clean (chongjonghan), dense (chomchomhan), dark, comfortable (kwaejeokhan), clunky, special, blue (pureureun), soft (puksinhan), abundant (pungbuhan), fragrant, bluff, vibrant, thrilling (hwangholhan), joy, difficult, rare (huigwihan), anthemic, fine, sturdy, sublime, mellow (geueukhan), thrilling (heungbundoeneun), infinite, venturesome' (Table 1).

The result of connecting the forest sensibility vocabulary with the Korean adjective classification system by Sin (1996) and Hong and Jeong (2009) is as follows. In the subcategory of sense vocabulary, words of 'color (green/blue), brightness (bright), purity (clear, clean)' were extracted from sight. 'Silent' was extracted from hearing and 'fragrant' from smell. Words of 'touch (soft, moist), temperature (warm, cool)' were extracted from touch. In the subcategory of evaluation vocabulary, 'noble or lowly (valuable), beauty or ugliness (beautiful, nice, natural), profit (safe), comparison (special)' were extracted. In the subcategory of existence vocabulary, 'depth (deep), density (dense)' were extracted from space, 'high or low (high), size (big), weight (light), balance (even, rugged)' were extracted from shape, and 'many (various), plentiful' were extracted from quantity. In emotion vocabulary, 'joy and sorrow/anger (glad, excited, fresh), preference (good), satisfaction/dissatisfaction (relaxed), fear/trust (familiar, comfortable)' were extracted, and 'pleasure (rewarding, rapture, happy, sweet, cozy), gratitude (grateful), acceptance (fluttering, kind)' were added by supplementing emotion verbs of Hong and Jeong (2009). Out of total 137 words, 25 failed to be included in the Korean classification system. To decide whether these words are suitable for forest sensibility vocabulary, a Delphi survey was conducted on forest experts. The results showed that 'sweet, grateful, kind, moist, cozy, glad, special, light, rugged' were included in the sense, emotion, evaluation, and existence category of the Korean classification system. We developed the forest sensibility vocabulary classification system comprising of four categories, 25 subcategories and 40 sensibility words throughout the series of processes (Figure 3; Table 2). 
Table 1. List of 137 sensibility words relating to forest welfare extracted from magazines, books, and written experiences

\begin{tabular}{|c|c|c|c|c|c|c|c|}
\hline No & Vocabulary & No & Vocabulary & No & Vocabulary & No & Vocabulary \\
\hline 1 & Good & 36 & Refreshed & 71 & Recover & 106 & Hefty \\
\hline 2 & Comfortable (Pyeonanhan) & 37 & Various & 72 & Mellow (Gammiroun) & 107 & Harmonious \\
\hline 3 & Beautiful (Areumdaun) & 38 & Lush & 73 & Appreciation & 108 & Rare (Jingwihan) \\
\hline 4 & Clean (Malgeun) & 39 & Calm (Chabunhan) & 74 & Modesty & 109 & Subside \\
\hline 5 & Happiness & 40 & Peaceful (Pyeonganhan) & 75 & Arduous & 110 & Grumous \\
\hline 6 & Fresh (Sang Kwaehan) & 41 & Quiet (Pyeongonhan) & 76 & Straight & 111 & Racy \\
\hline 7 & Stabile & 42 & Interesting & 77 & Great (Goengjanghan) & 112 & Sunshiny \\
\hline 8 & Enjoyable & 43 & Glad & 78 & Romantic & 113 & Liquid \\
\hline 9 & Dense (Ulchanghan) & 44 & Soft (Budeureoun) & 79 & Unfamiliar & 114 & Clean (Chongjonghan) \\
\hline 10 & Blue (Pureun) & 45 & New & 80 & High & 115 & Dense (Chomchomhan) \\
\hline 11 & Arcane & 46 & Safety & 81 & Varied & 116 & Dark \\
\hline 12 & Cool & 47 & Jeonggyeoun & 82 & Great (Daedanhan) & 117 & Comfortable (Kwaejeokan) \\
\hline 13 & Warm (Ttatteutan) & 48 & Moist & 83 & Warm (Ttaseuhan) & 118 & Clunky \\
\hline 14 & Excited & 49 & Abundant (Pungseonghan) & 84 & Tremble & 119 & Special \\
\hline 15 & Fresh (Singgeureoun) & 50 & Convenient & 85 & Treach & 120 & Blue (Pureureun) \\
\hline 16 & Leisurely & 51 & Unchangeable & 86 & Soft(Bodeuraun) & 121 & Soft (Puksinhan) \\
\hline 17 & Thankful & 52 & Old & 87 & Fruitful & 122 & Abundant (Pungbuhan) \\
\hline 18 & Valuable & 53 & Light & 88 & Full & 123 & Fragrant \\
\hline 19 & Tough & 54 & Deep & 89 & Fresh (Santteuhan) & 124 & Bluff \\
\hline 20 & Wonderful & 55 & Amazing (Nollaun) & 90 & Animated & 125 & Vibrant \\
\hline 21 & Refreshing & 56 & Dazzling & 91 & Beautiful (Suryeohan) & 126 & Thrilling (Hwangholhan) \\
\hline 22 & Pretty & 57 & Friendly & 92 & Amazing (Singihan) & 127 & Joy \\
\hline 23 & Clear & 58 & Nice & 93 & Charming & 128 & Difficult \\
\hline 24 & Clean (KKaekkeutan) & 59 & Bright & 94 & Snug (Aneukan) & 129 & Rare (Huigwihan) \\
\hline 25 & Attractive & 60 & Inconvenient & 95 & Dizzy & 130 & Anthemic \\
\hline 26 & Flutter & 61 & An armful & 96 & Comfort & 131 & Fine \\
\hline 27 & Snug (Pogeunhan) & 62 & Grand & 97 & Colorful & 132 & Sturdy \\
\hline 28 & Abundant (Pungyoroun) & 63 & Great (Widaehan) & 98 & Bright & 133 & Sublime \\
\hline 29 & Healthy & 64 & Delicate & 99 & Demulcent & 134 & Mellow (Geueukan) \\
\hline 30 & Silence & 65 & Cold (Chagaun) & 100 & Pleasant & \multicolumn{2}{|c|}{135 Thrilling (Heungbundoeneun) } \\
\hline 31 & Dense (Ppaekppaekan) & 66 & Cosy & 101 & Familiar & 136 & Infinite \\
\hline 32 & Funny & 67 & Big & 102 & Fine & 137 & Venturesome \\
\hline 33 & Silent & 68 & Peace & 103 & Natural & & \\
\hline 34 & Peaceful (Pyeonghwaroun) & 69 & Warm (Pugeunhan) & 104 & Free & & \\
\hline 35 & Fancy & 70 & Quiet (Pugeunhan) & 105 & Calm (Janjanhan) & & \\
\hline
\end{tabular}




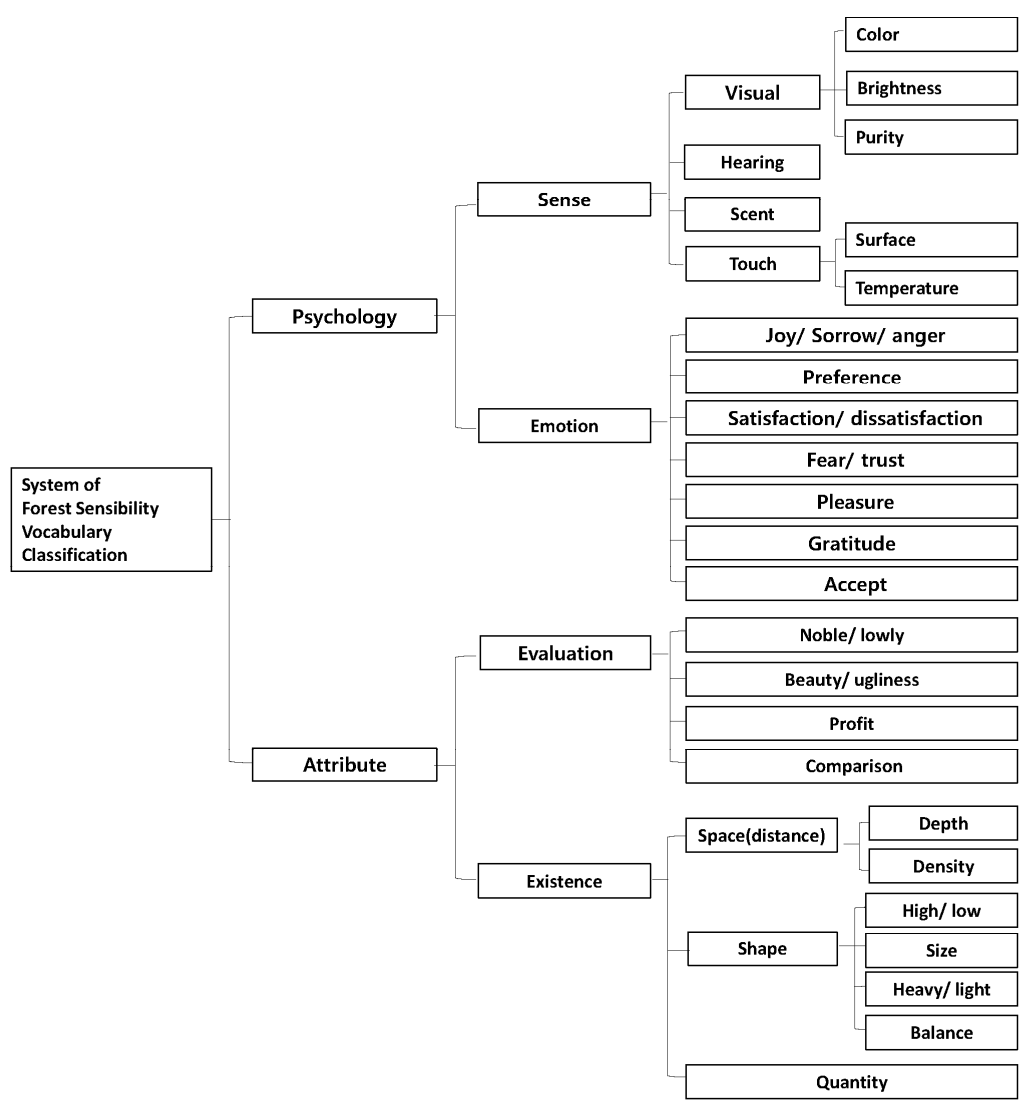

Figure 3. Development of forest sensibility vocabulary classification system.

Table 2. Contents of gardening activities in the program

\begin{tabular}{|c|c|c|c|}
\hline Category & \multicolumn{2}{|c|}{ Subcategory } & Sensibility vocabulary \\
\hline \multirow{7}{*}{ Sense vocabulary } & \multirow{3}{*}{ Visual vocabulary } & Color & Green \\
\hline & & Brightness & Bright \\
\hline & & Purity & Clear, clean \\
\hline & \multirow{2}{*}{\multicolumn{2}{|c|}{$\begin{array}{l}\text { Hearing vocabulary } \\
\text { Scent vocabulary }\end{array}$}} & Silent \\
\hline & & & Fragrant \\
\hline & \multirow{2}{*}{ Touch vocabulary } & Surface & Soft, moist \\
\hline & & Temperature & Warm, cool \\
\hline \multirow{7}{*}{ Emotion vocabulary } & \multicolumn{2}{|c|}{ Joy/sorrow, anger } & Glad, excited, fresh, \\
\hline & \multicolumn{2}{|c|}{ Preference } & Good \\
\hline & \multicolumn{2}{|c|}{ Satisfaction/dissatisfaction } & Relaxed \\
\hline & \multicolumn{2}{|c|}{ Fear/trust } & Familiar, comfortable \\
\hline & \multicolumn{2}{|c|}{ Pleasure } & Rewarding, rapture, happy, sweet, cozy \\
\hline & \multicolumn{2}{|c|}{ Gratitude } & Grateful \\
\hline & \multicolumn{2}{|c|}{ Accept } & Flutter, kind \\
\hline \multirow{4}{*}{ Evaluation vocabulary } & \multicolumn{2}{|c|}{ Noble/lowly } & Valuable \\
\hline & \multirow{2}{*}{\multicolumn{2}{|c|}{$\begin{array}{l}\text { Beauty/ugliness } \\
\text { Profit }\end{array}$}} & Beautiful, nice, natural \\
\hline & & & Safety \\
\hline & \multicolumn{2}{|c|}{ Comparison } & Special \\
\hline \multirow{7}{*}{ Existence vocabulary } & \multirow{2}{*}{ Space (distance) } & Depth & Deep \\
\hline & & Density & Dense \\
\hline & \multirow{4}{*}{ Shape } & High/low & High \\
\hline & & Size & Big \\
\hline & & Heavy/light & Light \\
\hline & & Balance & Even, rugged \\
\hline & \multicolumn{2}{|c|}{ Quantity } & Many (various), plentiful \\
\hline
\end{tabular}




\section{Conclusion}

Total 40 words were extracted in the developed forest sensibility vocabulary classification system, which can be largely classified into psychology and attributes according to the subject. Words according to the psychological factors of humans are generally influenced by the internal and external aspects of humans, thereby depicting sensibility words. Words mostly expressing shapes or characteristics such as attributes depict sensibility words shown as they are by the object. Sense and emotion words are those concentrated on human psychology, and evaluation and existence words are those concentrated on attributes such as characteristics or fundamental properties of objects. More specifically, the dictionary definition of sense is the phenomenon of consciousness that occur when external stimulation and pain such as light or sound are transmitted to the central nerves (Sense, n.d.). Sense vocabulary is influenced by 'sight, hearing, smell, touch' among the five senses felt by visitors through the forest environment, but did not show taste, implying that it may not have much influence in forest sensibility. Next, the dictionary definition of emotion is the feeling or mood that occurs in mind when encountering a certain phenomenon or event, generally referring to joy, anger, sorry and pleasure, comfort/discomfort, satisfaction and fear (Emotion, n.d.). Emotion vocabulary expresses 'joy and sorrow/anger, preference, satisfaction, fear/trust, gratitude,' with positive influence such as 'glad, relaxed, comfortable, fluttering, happy.' Next, there are various dictionary definitions of evaluation, but in this study it means to determine the value of a certain phenomenon or object, including not only the good and evil or beauty and ugliness or humans but also the value of objects (Evaluation. n.d.; Sin, 1996). Evaluation vocabulary mostly expresses 'beautiful, nice, valuable, natural, safe, special' such as 'noble or lowly, beauty or ugliness, profit, comparison,' but did not show 'authenticity (true or false), right or wrong, good or evil difficulty (easy or difficult),' implying that these words may not have much influence in forest sensibility. Finally, the dictionary definition of existence is everything that can be considered as 'existing,' representing objective behaviors or special properties of humans or objects (Existence, n.d.; Sin, 1996). Existence vocabulary expresses 'deep, dense, high, big, light, straight, various, abundant' such as 'space, shape, quantity,' but did not show 'time, distance, weight, diligence, privacy,' implying that these words may not have much influence in forest sensibility. Finally, most words excluding emotion words primarily accept information through sight, and thus form or shape of forest landscape may have served as a critical factor in sensibility expression (Lee, 2007). This is because there is a high ratio of words influenced by sight in sense, evaluation, and existence vocabulary. For example, 'green/blue, bright, clear' in sense vocabulary, 'dense, abundant, various, deep, high, straight, big, light, rugged' in existence vocabulary,' and 'nice, beautiful, natural' in evaluation vocabulary are all expressions influenced by sight.

The objective of this study is to develop a forest sensibility vocabulary classification system for sensibility evaluation of users in the forest environment. We extracted forest sensibility vocabulary from countless sensibility words and intended to determine which factors affect sensibility experienced by forest visitors with discrimination and objectivity. It seems that additional research that connects forests with sensibility must be conducted in order to increase qualitative satisfaction in forest leisure and welfare. The results of this study are expected to be used as data about the forest environment and human sensibility.

\section{References}

Choi, H.B. 1937. Urimalbon [Basic Korean]. Seoul, Korea: Jungeumsa.

Emotion. n.d. In Doopedia. Retrieved November 14, 2018 from http://www.doopedia.co.kr

Evaluation. n.d. In Educational Psychology Glossary. Retrieved November 14, 2018 from

https://terms.naver.com/entry.nhn?docId=1944941\&cid=41989\&categoryId=41989 
Existence. n.d. In Doopedia. Retrieved November 14, 2018 from http://www.doopedia.co.kr

Hong, J.S. and Y.J. Jeong. 2009. Establishing the category of emotion verb and classifying emotion verbs. Korean Linguist. 45:387-420.

Hong, S.M. 1985. A study on the meaning of Korean vocabulary. Seoul, Korea: Hakmunsa.

Jang, H.J. 2001. Classification system for emotional verbs and adjectives. Proc. Conf. Korean Soc. Inf. Manag. 8:29-34.

Jung, H.W. and K. Nah. 2007. A study on the meaning of sensibility and vocabulary system for sensibility evaluation. J. Ergono. Soc. Korea 26(3):17-25.

Korea Forest Service. 2012. Development and utilization of forest grade evaluation method (pp. 14-16). Daejeon, Korea: Author.

Korea Forest Service. 2014. Forest Healing Experience Water Prize Collection. Daejeon, Korea: Author.

Korea Forest Service. 2014-2015. Eco Healing 1-7 Issue. Daejeon, Korea: Author.

Korea Forest Service. 2017. Statistical yearbook of forestry (pp. 337-340). Daejeon, Korea: Author.

Korea Forest Welfare Institute. 2017. Mid- and long-term management goals (pp. 19-30). Daejeon, Korea: Author.

Lee, C.M. 1976. Cases for psychological verbs in Korean. Korean J. Linguist. 1(1):256-296.

Lee, J.D., C.H. Park, D.W. Joung, S.M. Koo, and B.J. Park. 2019. Components of phytoncide from a pine forest in the southern temperate zone. Korean J. Agric. Sci. 46(1):33-34.

Lee, J.S. and H.N. Kim. 2013. A study on Image Analysis Model for exterior color of apartments using Multidimensional Scaling Method. J. Korean Soc. Color Stud. 27(1):133-141.

Lee, K.H. 1998. Human sensibility and emotion in sensibility ergonomics. Korean J. Sci. Emot. Sensib. 1(1):113-122.

Lee, M.Y. and H.C. Lee. 1990. The structural analysis of adjective meanings: Related to affective vocabulary. Korean J. Exp. Cogn. Psychol. 2:118-138.

Lee, Y.H. 2007. A study on the landscape management methods by the characteristics of the visual preference of forest landscape - Focused on Korea National Arboretum -. Master's thesis, Kookmin University, Seoul, Korea.

Nam, K.S. and Y.G. Ko. 1985. Standard Korean grammar. Seoul, Korea: Top Publishing.

Sense. n.d. In Doopedia. Retrieved November 14, 2018 from http://www.doopedia.co.kr

Sin, S.J. 1996. Classification of Adjectives Language Collection of Dissertations. Doctoral dissertation, Sookmyung Women's University, Seoul, Korea.

Sohn, S., M.S. Park, J.E. Park, and J.H. Sohn. 2012. Korean emotion vocabulry: Extraction and categorization of feeling words. Korean J. Sci. Emot. Sensib. 15(1):105-120.

Son, Y.J. 1992. Classification system of sensory adjectives. Journal of the Daegu Languagen. 10:127-154. Retrieved from http://www.urimalgeul.org/sobis/uirm.jsp

Standard Korean Dictionary. 2018. Retrieved from http://stdweb2.korean.go.kr/main.jsp

Yoo, H.K. 2000. A study on the classification of Korean adjectives. J. Korean Linguist. 36:221-258. 